\title{
F-Wave Waveform Values Based on the Correlation Coefficient of Each Waveform Increased Following Improved Voluntary Movements in a Patient with Cerebrovascular Disease: A Case Study with Long-term Follow-up
}

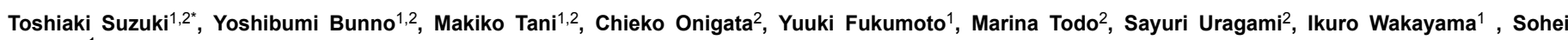
Yoshida $^{1}$

${ }^{1}$ Graduate School of Health Sciences, Graduate School of Kansai University of Health Sciences

${ }^{2}$ Clinical Physical Therapy Laboratory, Faculty of Health Sciences, Kansai University of Health Sciences

*Corresponding author: Toshiaki Suzuki, Graduate School of Health Sciences, Graduate School of Kansai University of Health Sciences, 2-11-1, Wakaba, Kumatori, Sennan, Osaka 590-0482, Japan, Tel:+81724538374; Fax: +81724538798; E-mail: suzuki@kansai.ac.jp

Rec date: April 21, 2016; Acc date: April 27, 2016; Pub date: April 30, 2016

Copyright: (C) 2016 Suzuki T, et al. This is an open-access article distributed under the terms of the Creative Commons Attribution License, which permits unrestricted use, distribution, and reproduction in any medium, provided the original author and source are credited.

\begin{abstract}
To investigate the effect of physical therapy on F-wave characteristics in a patient with cerebrovascular disease, we tested the relationship between F-wave waveforms and the recovery of voluntary movement on the affected side. We measured F-waves of affected thenar muscles after stimulating the affected median nerve at the wrist in a left hemiplegia patient (female, 60 years old) with cerebrovascular disease. We analysed the F/M amplitude ratio and Fwave waveform values from 30 trials.

F-wave waveform values were determined as follows. We calculated the correlation coefficient of each waveform and defined identifying F-waves as those with a correlation coefficient greater than 0.9 . We determined the number of different wave forms from the number of identifying waveforms. F-waves were tested three times at 9,52 , and 70 months from sideration. The patient underwent physical therapy twice a week, with each session lasting 20 min. The F/M amplitude ratio gradually decreased and the number of F-wave waveform types gradually increased. Muscle tonus and voluntary movements of the affected arm gradually improved with physical therapy. In this patient with cerebrovascular disease, F-wave waveform values in her affected arm gradually increased as muscle tonus and voluntary movements of the arm improved.
\end{abstract}

\section{Introduction}

An F-wave is the result of the backfire of $\alpha$-motor neurons following an antidromic invasion of propagated impulses across the axon hillock [1]. Its occurrence reflects excitability changes in the spinal motor neurons, as reported in patients with spasticity [2]. In an earlier study that investigated the nervous system of hemiplegic patients, we evaluated the excitability of spinal neural function using the F-wave data of patients with cerebrovascular disease (CVD) [3]. We also reported that the $\mathrm{F}$-wave persistence and $\mathrm{F} / \mathrm{M}$ amplitude ratio in patients with CVD were affected by the grade of muscle tonus, tendon reflex, and voluntary movement. It has been reported that persistence depends on the number of neuromuscular units activated, whereas the $\mathrm{F} / \mathrm{M}$ amplitude ratio depends on their excitability [4]. We therefore concluded that F-wave measurement was an effective neurological test for evaluating muscle tonus and voluntary movements. The characteristics of F-wave waveforms differ between supramaximal stimulations. However, we found that supramaximal stimulation in CVD patients with hypertonus always evoked the same waveform, similar to the H-reflex. In this study, we investigated the effect of physical therapy with long-term follow-up in a CVD patient with hypertonus of her affected arm by measuring F-wave parameters such as persistence, $\mathrm{F} / \mathrm{M}$ amplitude ratio and $\mathrm{F}$-wave waveform, and voluntary movement recovery on the affected side.

\section{Materials and Methods}

\section{Subject}

The patient was a 60 -year-old female with left-sided hemiplegia caused by CVD. She had hypertonus of thenar muscles and difficulty in moving the thenar in the first F-wave test, 9 months after consideration. Written informed consent was obtained from the subject. This study was approved by the Research Ethics Committee at Kansai University of Health Sciences. The experiments were conducted in accordance with the Declaration of Helsinki.

\section{The F-wave tests}

The subject was maintained comfortably in a supine position with external rotation of both shoulder joints. The skin was prepared with abrasive gel to maintain the impedance below $5 \mathrm{k} \Omega$. A Viking Quest electromyography machine (Natus Medical Inc., CA, and USA) was used to record the F-waves. A pair of round disks was attached with collodion to the skin of the left thenar muscles over the muscle belly and the bone of the metacarpophalangeal joint of the thumb. After stimulating the left median nerve at the wrist during relaxation, we tested the F-wave. The stimulating electrodes comprised a cathode placed over the left median nerve at $3 \mathrm{~cm}$ proximal to the palmar crease of the wrist joint and an anode placed $2 \mathrm{~cm}$ further proximally. The maximal stimulus was determined by delivering $0.2 \mathrm{~ms}$ squarewave pulses of intensity sufficient to elicit the largest compound muscle 
Citation: Suzuki T, Bunno Y, Tani M, Onigata C, Fukumoto Y, et al. (2016) F-Wave Waveform Values Based on the Correlation Coefficient of Each Waveform Increased Following Improved Voluntary Movements in a Patient with Cerebrovascular Disease: A Case Study with Long-term Follow-up. Int J Neurorehabilitation 3: 206. doi:10.4172/2376-0281.1000206

Page 2 of 4

action potentials. To acquire the F-waves, 30 supramaximal shocks (adjusted up to a value $20 \%$ higher than the maximal stimulus) were delivered at $0.5 \mathrm{~Hz}$. The bandwidth filter ranged from $5 \mathrm{~Hz}$ to $2 \mathrm{kHz}$.

\section{Data analysis}

We analyzed the persistence, $\mathrm{F} / \mathrm{M}$ amplitude ratio, and $\mathrm{F}$-wave waveform values from 30 trials. Persistence was defined by the number of measurable F-wave responses divided by 30 trials of supramaximal stimulation. The $\mathrm{F} / \mathrm{M}$ amplitude ratio was defined as the mean amplitude of all the measurable responses divided by the amplitude of the $\mathrm{M}$-wave. $\mathrm{F}$-wave waveform values were determined as follows. The window from minimum to maximum onset latency was determined from the measurable F-waves. Next, we calculated the correlation coefficient of each waveform using Microsoft Excel, and defined identifying $\mathrm{F}$-waves as those with a correlation coefficient of $>0.9$. Finally, we determined the number of different waveforms from the number of identifying waveforms. F-waves were tested three times at 9 , 52 , and 70 months from sideration. The patient underwent physical therapy twice a week, with each session lasting $20 \mathrm{~min}$. The aim of the physical therapy was to improve the muscle tone of the thinner muscles and to facilitate extension movement of the patient's digits, especially the thumb.

\section{Results}

The F/M amplitude ratio gradually decreased from $7.67 \%$ at 9 months from sideration to $4.12 \%$ at 52 months and $2.07 \%$ at 70 months. At the same time, muscle tonus of the digits, especially the thumb, also improved (Figure 1). The F-wave waveform values changed as follows. At 9 months after sideration, there were only six types of F-wave waveforms. An F-wave waveform (Figures 2 and 3) almost appeared. At 52 months after sideration, there were again six types of F-wave waveform. However, the six waveform types now appeared equally (Figures 4 and 5). At 70 months after sideration, the number of F-wave waveform types increased to 16 (Figures 6 and 7).

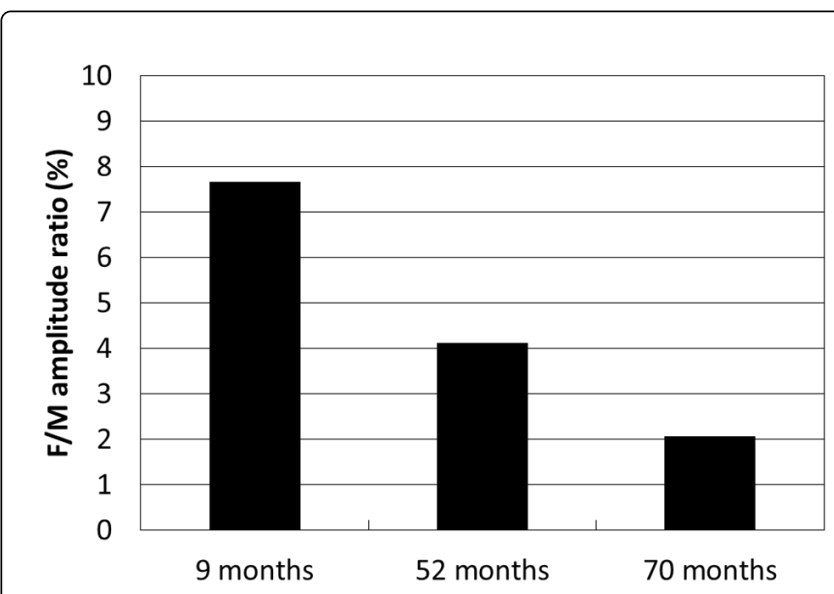

Figure 1: The $\mathrm{F} / \mathrm{M}$ amplitude ratio at 9,52 , and 70 months from consideration.

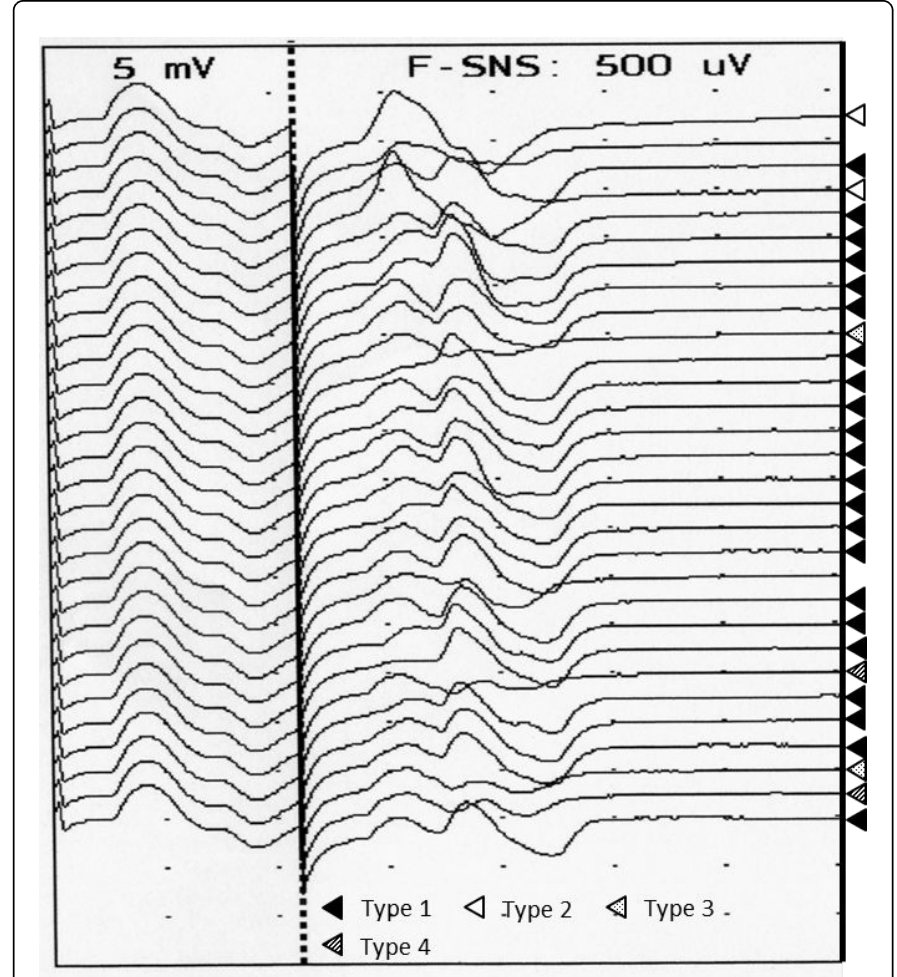

Figure 2: The F-wave waveform at 9 months after consideration.

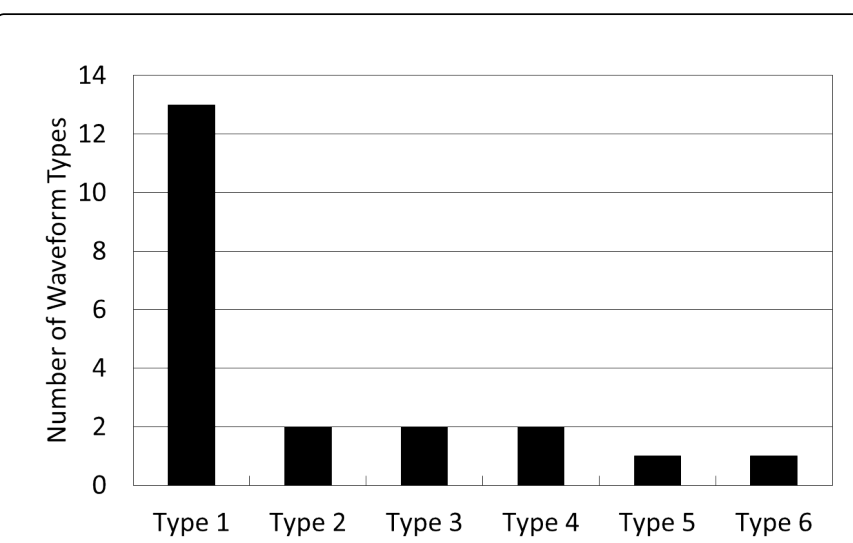

Figure 3: The number of F-wave waveform types at 9 months after sideration.

The following specific functional changes occurred in the patient's affected hand. At 9 months after sideration, movement of the digits on the affected side was poor; total flexion of the fingers was possible but extension was difficult; and, in particular, she was unable to move her thumb. At 52 months after sideration, digit movement on the affected side was moderate; total flexion and extension of fingers was possible, but extension of the thumb was poor. At 70 months after sideration, finger movement on the affected side was excellent; separate movements of the fingers had improved; and, in particular, thumb movement was also good. 
Citation: Suzuki T, Bunno Y, Tani M, Onigata C, Fukumoto Y, et al. (2016) F-Wave Waveform Values Based on the Correlation Coefficient of Each Waveform Increased Following Improved Voluntary Movements in a Patient with Cerebrovascular Disease: A Case Study with Long-term Follow-up. Int J Neurorehabilitation 3: 206. doi:10.4172/2376-0281.1000206

Page 3 of 4

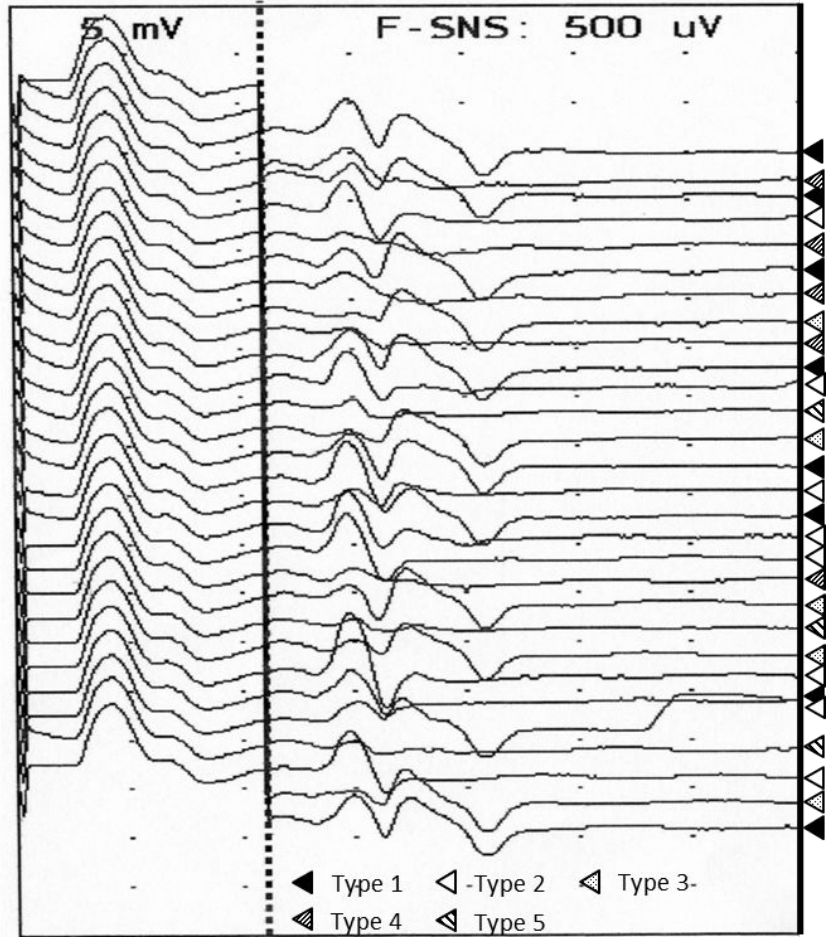

Figure 4: The F-wave waveform at 52 months from sideration.

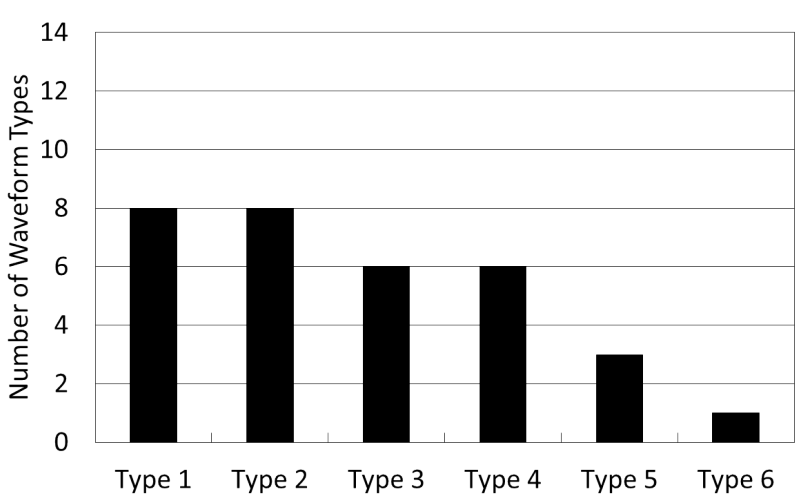

Figure 5: Number of F-wave waveform types at 52 months after sideration.

\section{Discussion}

At 9 months after sideration, almost every F-wave had the same pattern, similar to the H-reflex. However, we considered this patient's waveforms to be F-waves because F-wave was recorded follow $\mathrm{M}$-wave. She was not able to move her affected thumb voluntarily due to hypertonus of the affected thumb muscles. There were six types of Fwave waveform at this time, with one type of waveform appearing many times.

After physical therapy, the patient's F-wave waveforms became more varied, and she regained physical skill in her affected thinner muscles.
The reasons for this change were as follows. At the start of physical therapy, her F-waves were almost the entire same pattern; this was because we gave electrical stimulation to the median nerve to produce the F-wave, when the excited $\alpha$-motor neurons were similar. After the physical therapy had been effective and the physical skill of the affected thumb had improved, various $\alpha$-motor neurons could be excited.

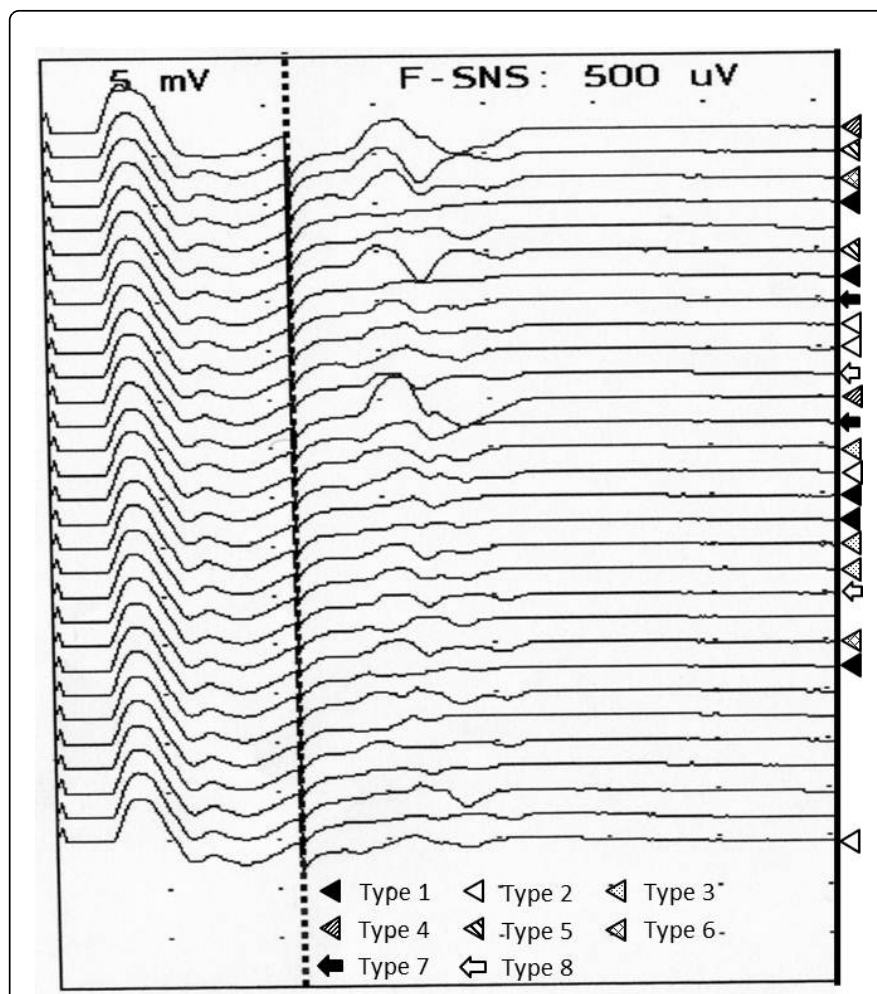

Figure 6: The F-wave waveform at 70 months after sideration.

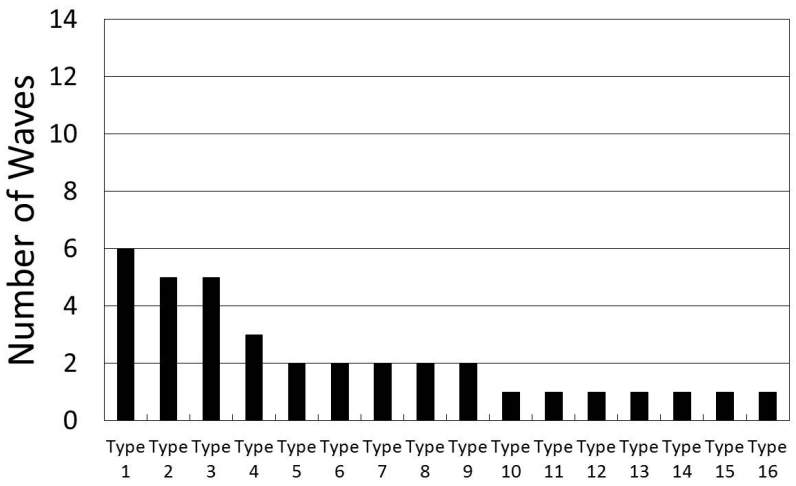

Figure 7: The number of F-wave waveform types at 70 months after sideration. Digit movement in the affected side improved.

It is believed that this change was due to an increase in the number of anterior horn cells, which compose F-waves, as voluntary movement on the affected side recovered (Figure 8). 
Citation: Suzuki T, Bunno Y, Tani M, Onigata C, Fukumoto Y, et al. (2016) F-Wave Waveform Values Based on the Correlation Coefficient of Each Waveform Increased Following Improved Voluntary Movements in a Patient with Cerebrovascular Disease: A Case Study with Long-term Follow-up. Int J Neurorehabilitation 3: 206. doi:10.4172/2376-0281.1000206

Page 4 of 4

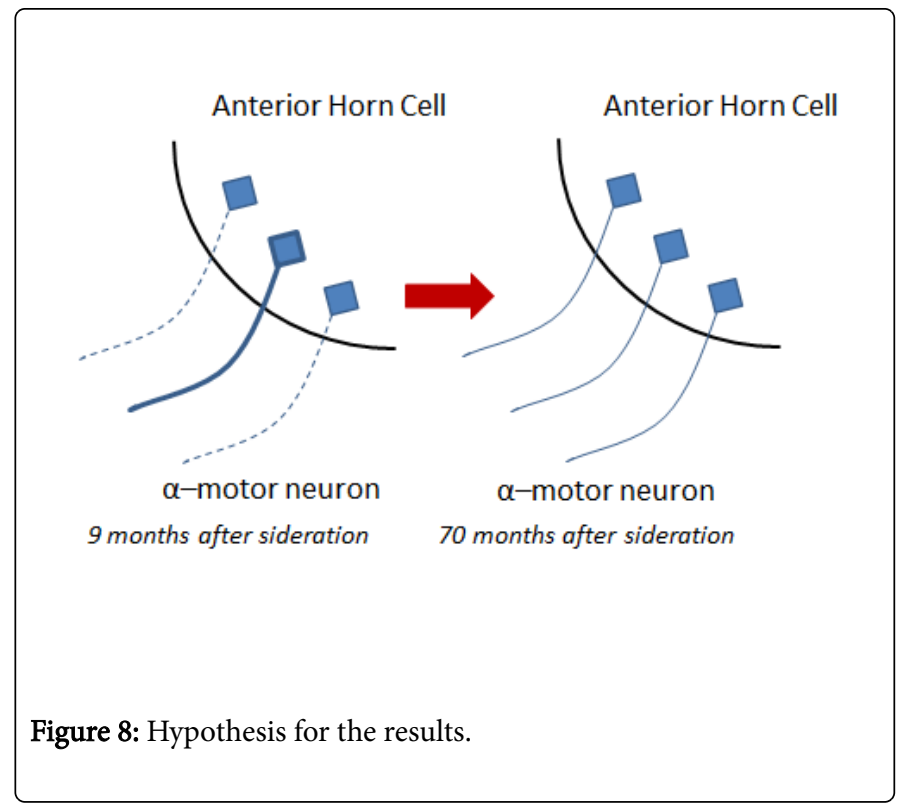

Till date, there have been no reports in patients with CVD of F-wave waveforms exhibiting many variations following effective physical therapy. The present study included only one patient, but in future, we aim to treat more patients with CVD by physical therapy and to try to confirm the relationship between the F-wave waveforms and the physical skill level of the patients.

\section{Conclusion}

In this patient with CVD, the F-wave waveform values in the arm gradually increased and improved as muscle tonus and voluntary movements of the affected arm improved.

\section{Conflicts of interest}

The authors declare that there were no conflicts of interest in this study.

\section{References}

1. Kimura J (1974) F-wave velocity in the central segment of the median and ulnar nerves. A study in normal subjects and in patients with CharcotMarie-Tooth disease Neurology 24: 539-546.

2. Suzuki T, Fujiwara T, Takeda I (1993) Characteristics of F-wave during relaxation in patients with CVD (In Japanese). PT journal 27: 277-281.

3. Eisen A, Odusote K (1979) Amplitude of the F wave: A potential means of documenting spasticity. Neurology 29: 1306-1309.

4. Odusote K, Eisen A (1979) An electrophysiological quantitation of the cubital tunnel syndrome. The Canadian Journal of Neurological Sciences 6: 403-410. 